\title{
Análisis comparativo entre el Chocó Biogeográfico y otros lugares del mundo en relación con el uso de plantas medicinales para la cura y/o alivio de las afecciones ocasionadas por la mordedura de serpientes
}

\section{Comparative analysis between the Chocó Biogeography and elsewhere in connection with the use of medicinal plants for cure and/or relief of condition caused by snakebite}

\author{
Carlos Ariel Rentería Jiménez
}

\begin{abstract}
Resumen
Mediante entrevistas semiestructuradas a 23 curanderos, ysabedores ancestrales de ocho comunidades (Quibdó, Pacurita, Tutunendo, Alto Baudó, Bagadó, Cuenca del río San Bernardo Patía Norte, San José de Guare y Bajo Calima) del Chocó Biogeográfico colombiano, se registraron 70 etno-especies de plantas antiofidicas, pertenecientes a 35 familias botánicas, de estas sólo tres se incluye entre las criptógamas (helechos o pteridofitas). Las familias con el mayor número de especies representativas fueron: 10 Piperaceas, 8 Araceas, 7 Gesneriaceas, 6 Rubiaceas, 5 Aristolachias y 3 Asteraceas o compuestas. Se realizó una comparación entre estas especies y las reportadas en otros estudios realizados en el Chocó Biogeográfico colombiano usadas para la mordedura de serpientes, con las informadas en la literatura y utilizadas para el mismo fin en otros lugares del mundo. Siendo las Piperaceas la familia más representativa con 17 especies, seguido de la familia de las Araceas con 13 especies a nivel del Chocó Biogeográfico colombiano. En cuanto a los géneros el más representativo fue el Piper con 17 especies y los géneros Dracontium, Aristolochia y Columea con 4 especies cada uno. Se reportan tres nuevas etno-especies de plantas antiofidicas que son utilizadas exclusivamente por comunidades negras e indígenas del Chocó Biogeográfico colombiano: Tectaria vivipara, Costus vellosisumus, Adiantum raddianum.
\end{abstract}

Palabras clave: Plantas medicinales para el tratamiento de mordedura de serpientes; Sabedores ancestrales del Chocó Biogeográfico colombiano.

\begin{abstract}
By twenty-three semi-structured interviews healers, knowing and ancestral sabedoras? eight communities (Pretoria, Pacurita, Tutunendo, Alto Baudó, Bagadó, San Bernardo Cuenca Patia River North, San José de Guare and Bajo Calima) the Colombian Chocó Biogeography. There were seventy-ethno antiofidicas plant species belonging to 35 botanical families; of these only three are included among the Cryptogams (Ferns and Pteridophytes). The families with the largest number of representative species were: 10 Piperaceae, 8 Araceae, 7 Gesneriaceas, 6 Rubiaceae, 5 and 3 Aristolachias Asteraceae or Compositae. A comparison between these species and those reported in other studies in the Colombian Choco used for snakebites, with those reported in the literature and used for the same purpose in other parts of the world. Being the most representative Piperaceae family with 17 species, followed by the family
\end{abstract}

* Magister en Docencia de la química. Investigador de Proyectos Especiales del Instituto de Investigaciones Ambientales del Pacifico (IIAP), Quibdó, Colombia. e-mail: carlosariel.renteria@gmail.com 


\section{Comparación del uso de plantas medicinales para la cura y/o alivio por mordeduras de serpientes. CA. Rentería}

of the Araceae with 13 species at the Colombian Chocó. As for the most representative genres was the Piper with 17 species and genera Dracontium, Aristolochia and Columea with 4 species each. We report three new ethno-antiofidicas plant species that are used exclusively by black and indigenous communities in the Colombian Chocó: Tectaria viviparous, Costus vellosisumus, Adiantum raddianum.

Keywords: Medicinal plants to treat snakebite; Knowing and ancestral knowledgeable of Colombian biogeography Chocó.

\section{Introducción}

El uso de plantas medicinales constituye una tradición ancestral generalizada en todo el mundo, sobre todo en rituales mágico-religiosos (Firenzouli et al., 2005) o como medicamento (Li y Ohizumi, 2004). El empleo de productos naturales empíricos (ensayo y error) fue y continúa siendo, una de las formas habituales para buscar curación y alivio para enfermedades devastadoras, lesiones y males de la conceptualización popular. Estos aspectos constituyen los pilares fundamentales sobre los que se soportan los estudios etnobotánicos. Para la etnobotánica, documentar el uso de las plantas medicinales, en particular las usadas por comunidades negras e indígenas del Chocó Biogeográfico colombiano para contrarrestar los efectos producidos por la mordedura de serpientes venenosas, reviste suma importancia por el potencial que presentan en la aplicación etnofarmacológica. De igual manera, se busca contribuir al uso y aprovechamiento sustentable de los recursos naturales asociados con esta actividad, y su conocimiento, cuantificación e inventario constituyen el primer paso para el logro de este propósito (Tuxil y Nabhan, 1998; Martín, 2000), en especial en el caso de bosques tropicales en donde se recolectan varias especies.

Uno de los primeros trabajos de revisión sobre plantas medicinales reportadas en la literatura internacional como antídoto para la mordedura de serpientes es el realizado por Mors (1991) en el que se informa un número aproximado de 578 especies distribuidas en 94 familias botánicas. Una revisión posterior realizada por Houghton y Osibogun (1993) incrementa este número de plantas medicinales, estos autores registraron 781 especies, pertenecientes a 121 familias botánicas, pero es probable que este número sea bastante superior. En relación con la distribución geográfica, algunos países se destacan por su abundancia de plantas medicinales para el tratamiento de la mordedura de serpientes. En la india Mashkar y Caius (1931) y en Brasil Rizzini et al. (1988) reportan 311 y 100 especies, respectivamente. En México, el país con la mayor diversidad de especies de serpientes venenosas del continente americano (Campbell y Lamar, 1989), existen informes para 62 plantas antiofídicas (Noriega-
Trejos, 1993; Reyes et al., 1994, 1992).

A nivel nacional, Barranco-Pérezy Sánchez-Sáenz(2010), reporta con base en nombres vulgares un total de 72 plantas para controlar los efectos de la mordedura de distintas especies de serpientes usadas por médicos tradicionales en las estribaciones de la Sierra Nevada de Santa Marta.

A nivel regional, un excelente estudio sobre plantas del Chocó Biogeográfico utilizadas para el tratamiento de la mordedura de serpientes se realizó por un equipo de investigadores de las universidades de Antioquia y Chocó, coordinados por Rafael Otero. De este trabajo resulto el libro titulado «Plantas utilizadas contra mordedura de serpientes en Antioquia y Chocó, Colombia», el cual tiene información sobre el uso de 101 especies vegetales reportadas como antiofídicas por curanderos y chamanes (Otero, Fonnegra y Jiménez, 2000). Pese a la existencia de terapias adecuadas para el tratamiento de la mordedura de serpientes, cada año mueren en el mundo entre 35.000 y 40.000 personas. En el Chocó Biogeográfico la mayor incidencia de mordedura de serpientes ocurre en las zonas rurales, donde buena parte de su población pertenece a grupos étnicos agrupados en comunidades negras e indígenas, que en la mayoría de los casos relacionados con accidentes ofídicos solo dispone de los recursos terapéuticos que la selva proporciona, entre ellos, diversas plantas medicinales algunas de ellas con capacidad antiofídica a las que se le atribuye la capacidad de inhibir el envenenamiento, otras en cambio actúan como coadyuvantes, en especial para aliviar uno o varios síntomas complejos que se relacionan en casos de mordedura de serpientes venenosas, tales como fiebre, dolor, sangrado e inflamación.

De las cerca de 800 especies de plantas medicinales con propiedades antiofídicas que se conocen hoy en el mundo, la gran mayoría de los conocimientos que sobre ellas se tienen son esporádicos, accidentales y poco documentados. Se desconocen muchos aspectos de la medicina tradicional de innumerables grupos étnicos de todo el planeta; solo algunas han sido objeto de estudios científicos respecto a sus supuestas propiedades (Houghton y Osibogun, 1983). En el presente trabajo se revisan los hallazgos etnomedicinales sobre las plantas antiofídicas del Chocó Biogeográfico colombiano, también se abordan diversos aspectos relacionados con su etnobotánica y taxonomía.

\section{Metodología}

Área del estudio. La información etnobotánica fue recogida entre los años 2009 a 2011, en diferentes estudios realizados por el Instituto de Investigaciones Ambientales del Pacífico (IIAP) en diferentes sitios de su jurisdicción. El área geográfica comprendió los departamentos de Chocó, Cauca y Valle del Cauca. En Chocó, zona rural del municipio de Quibdó, corregimientos de Pacurita - 05 41' 00" N; $76^{\circ}$ 


\section{Bioetnia Volumen 9 № 1 (enero-junio), 2012}

$40^{\prime} 00^{\prime \prime} \mathrm{W}$ y Tutunendo - $05^{\circ} 44^{\prime} 41.7^{\prime \prime} \mathrm{N} ; 76^{\circ} 32^{\prime} 24.2^{\prime \prime} \mathrm{W}$,

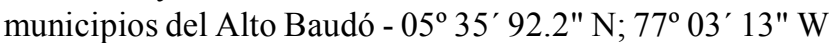
y Bagadó - 05 $24^{\prime} 32^{\prime \prime} \mathrm{N}$; $76^{\circ} 25^{\prime} 16^{\prime \prime} \mathrm{W}$; Cauca (norte del Cauca, la cuenca del río San Bernardo Patía Norte - 02 ${ }^{\circ} 30^{\prime}$

$00^{\prime \prime} \mathrm{N} ; 77^{\circ} 28^{\prime} 00^{\prime \prime}$ W y el corregimiento de San José de Guare $-02^{\circ} 33^{\prime} 23^{\prime \prime} \mathrm{N} ; 77^{\circ} 51^{\prime} 50^{\prime \prime} \mathrm{W}$; Valle del Cauca (zona de Bajo Calima en Buenaventura - 03 $55^{\prime} 00^{\prime \prime} \mathrm{N} ; 7^{\circ} 07^{\prime} 00^{\prime \prime}$ (Figura $1)$.

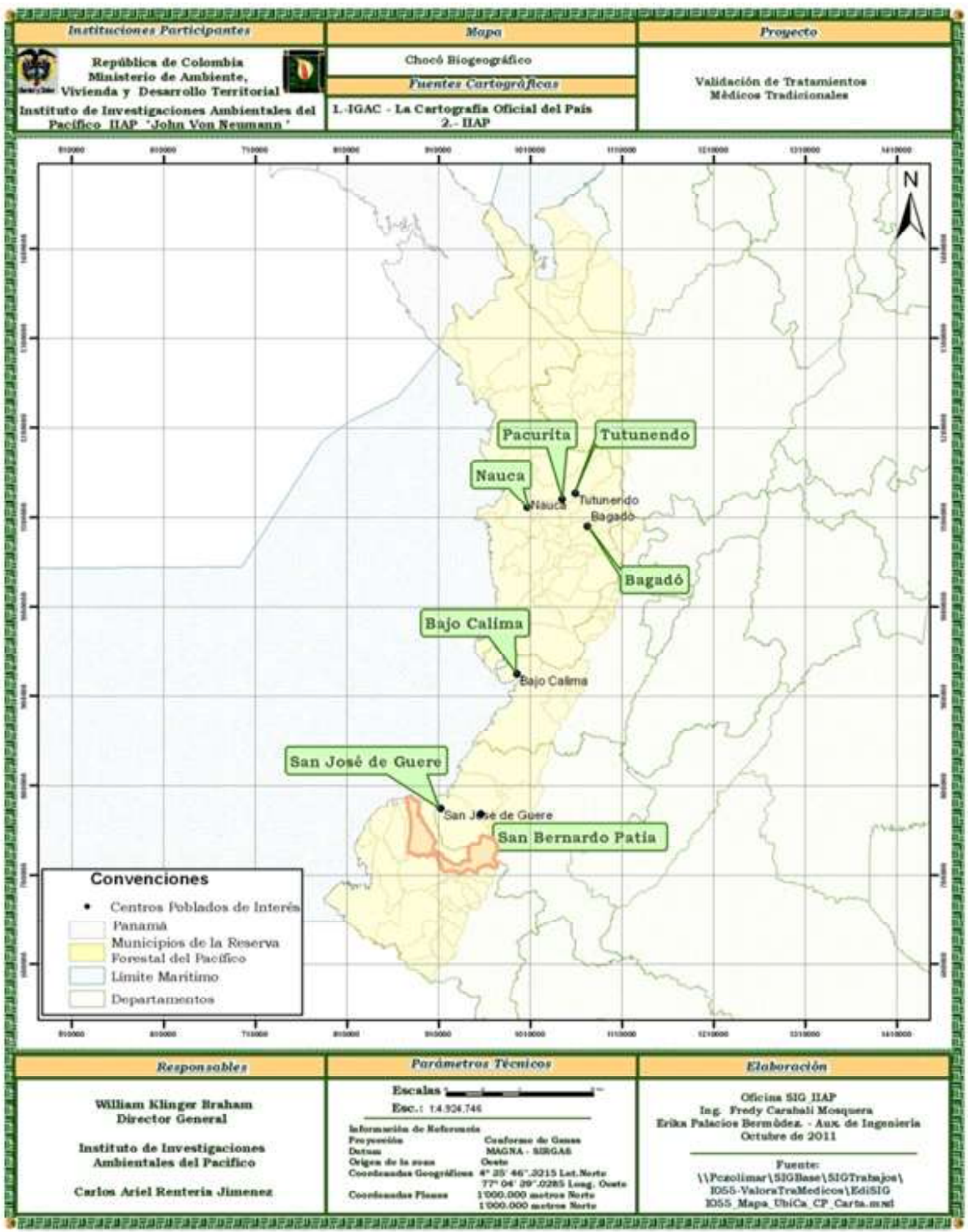

Figura 1. Mapa de localización del área de estudio 
Desarrollo metodológico. El desarrollo del estudio comprendió varias etapas articuladas una a otra; se inició con un proceso de socialización, al igual que se realizaba un proceso de profunda revisión de información secundaria, luego se registró y analizó la información etnobotánica en relación con las plantas medicinales utilizadas para el tratamiento de la mordedura de serpientes, trabajo que contó con la participación de importantes sabedores ancestrales de las comunidades donde se desarrolló este estudio y finalmente se realizó el documento final del cual se deriva el presente artículo científico.

\section{Registro y análisis de información etnobotánica}

Fuentes de información. La información colectada se logró a través de consultas a 23 informantes señalados por los habitantes de las ocho comunidades estudiadas como los mejores médicos tradicionales, sabedores del uso de plantas en casos de accidentes causados por mordedura de serpientes venenosas, a los cuales se les aplicó encuestas y entrevistas semiestructuradas, quienes a partir de sus experiencias como «curanderos» especializados en el tratamiento de diferentes casos de mordedura de serpientes, suministraron datos importantes relacionados con las plantas medicinales con acción terapéutica para el tratamiento y/o alivio de diferentes tipos de mordida de serpientes; la información tuvo como principal énfasis la identificación de diferentes aspectos relacionados con las características taxonómicas de las plantas medicinales usadas en las diferentes fórmulas tradicionales para el tratamiento de la mordedura de serpientes.

Identificación y descripción de las plantas medicinales. Se colectaron algunas de las plantas medicinales utilizadas por los médicos tradicionales para el tratamiento de la mordedura de serpientes y en otros casos descritas mediante revisión de literatura, adelantada por los investigadores en compañía de los médicos tradicionales, a fin de verificar el origen taxonómico, completar las descripciones botánicas y hacer una revisión bibliográfica exhaustiva de la especie o en su defecto el género y/o la familia. En este sentido el material botánico fue identificado por personal del Herbario de la Universidad Tecnológica del Chocó, en algunos casos partiendo de su nombre vulgar y haciendo la respectiva verificación de la muestra y en otros, se hizo por comparación directa de las muestras con material herbolario concentrado en su colección botánica.

Revisión de literatura. Se llevó a cabo una revisión de literatura en diferentes bases de datos como MEDLINE, LILACS, MAPA, NAPRALERT, ACULAR, ACUBOC, así como las bases cubanas CUMED Y METNA, al igual que consulta de documentos e informes generados por universidades y centros de investigación de Colombia, en busca de plantas medicinales reportadas como agentes antiofídicos.
Comparación de resultados. Con los resultados obtenidos en este estudio y los resultados de las consultas a las base de datos y revisión de documentos e informes generados por universidades y centros de investigación de Colombia en busca de plantas medicinales reportadas como agentes antiofídicos, se logró la identificación de las plantas antiofídicas reportadas en el Chocó Biogeográfico y en otros lugares del mundo, pudiéndose hacer comparaciones a nivel de familias, géneros y especies.

\section{Resultados y discusión}

La información se colectó con la ayuda de más de una veintena de médicos tradicionales, «curanderos» y jaibanás pertenecientes a las comunidades descritas en el área de estudio.

Principales plantas identificadas. En la Tabla 1 se muestra la información obtenida, basada en los nombres vulgares, donde se reportan 70 etno-especies utilizadas en diferentes tratamientos médicos tradicionales dirigidos a la cura y/o alivio de diferentes afecciones ocasionadas por la acción del veneno inoculado por mordedura de diferentes tipos de serpientes que habitan en el Chocó Biogeográfico colombiano.

Estas 70 etno-especies, pertenecen a 35 familias botánicas, de las que sólo tres se incluye entre las Criptogamas (helechos o pteridofitas), destacándose con el mayor número de especies representadas así: diez Piperaceas, ocho Araceas, siete Gesneriaceas, seis Rubiaceas, cinco Aristolachias, tres Asteraceas o compuestas. Por otro lado, las Cucurbitaceas, Simarubaceas y las Zingiberaceas tuvieron dos especies cada una; en el resto de las familias reportadas se tuvo un solo representante [Apocinaceas, Bixaceas, Cactaceas, Cesalpinaceas, Dryopteridaceas, Fabaceas (Leguminosas), Flacourtiaceas, Gencianaceas, Haemodoraceas, Lamiaceas, Lauraceas, Liliaceas, Loganiaceas, Malvaceas, Menispermaceas, Moraceas, Phytolaccaceas, Poligonaceas, Scrophulariaceas, Selaginelales, Ericaceas, Theophrastaceaes].

Estos datos contrastan con los reportados a nivel mundial, en donde se destaca que las familias botánicas con mayor número de especies antiofídicas son en su orden: Leguminosae y Asteraceae (7\% del total), Euphorbiaceae, Apocynaceae, Araceae, Aristolochiaceae y Verbenaceae (López y Pérez, 2009).

La familia Leguminosae se encuentra representada por 48 géneros y 70 especies de uso antiofídico a nivel mundial, que contrasta con solo 2 géneros y 3 especies de esta familia reportadas en el Chocó Biogeográfico como Bauhinia splendens, B. forficata y Desmodium triflorum. Las Asteraceas o compuestas representadas a nivel del Chocó Biogeográfico con 7 géneros (Mikania, Neurolaena, Wedelia, Adenosmma, Clibadium, Erechtites y Pseudoelephantopus) y 8 especies 
Bioetnia Volumen 9 № 1 (enero-junio), 2012

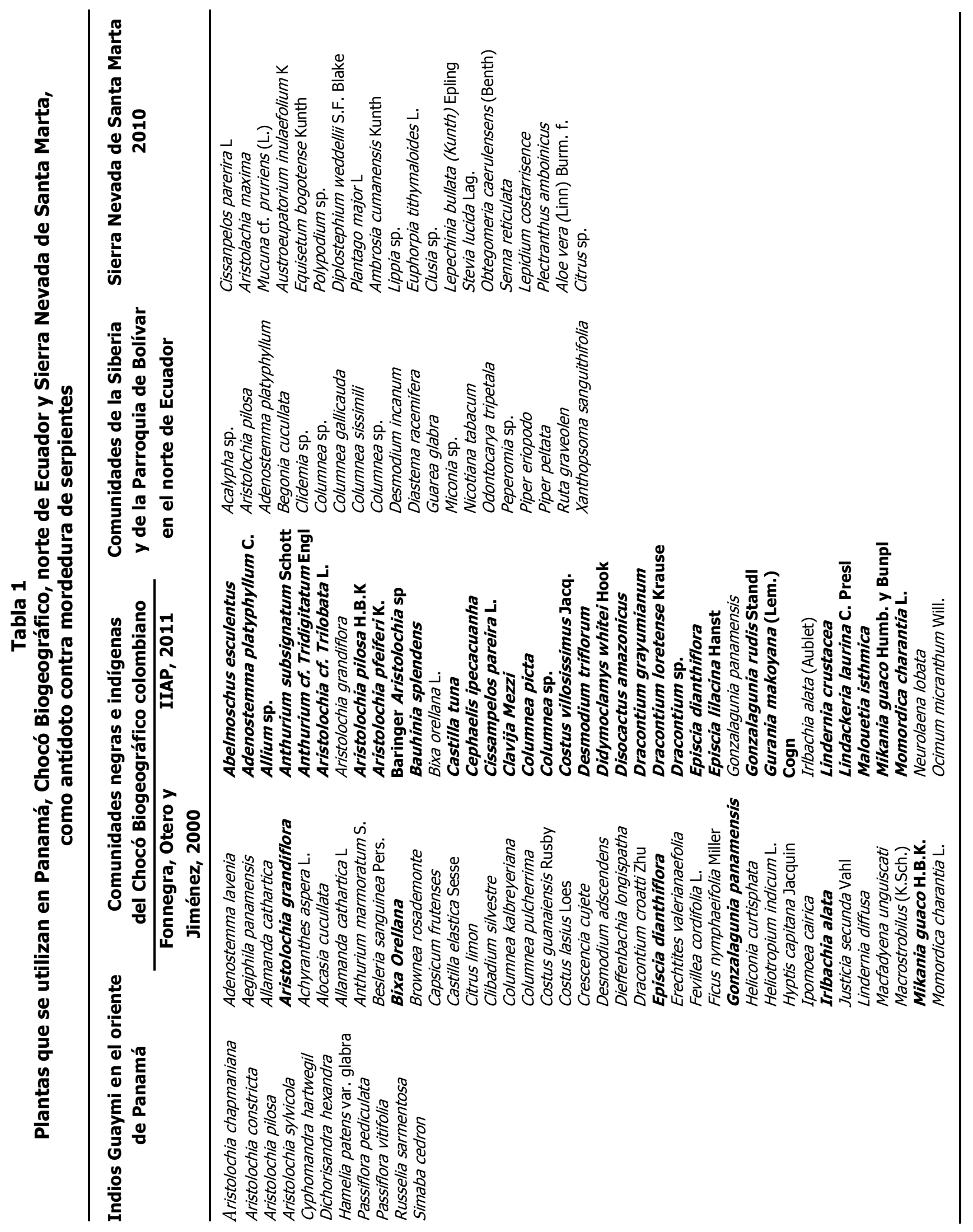


Comparación del uso de plantas medicinales para la cura ylo alivio por mordeduras de serpientes. CA. Rentería

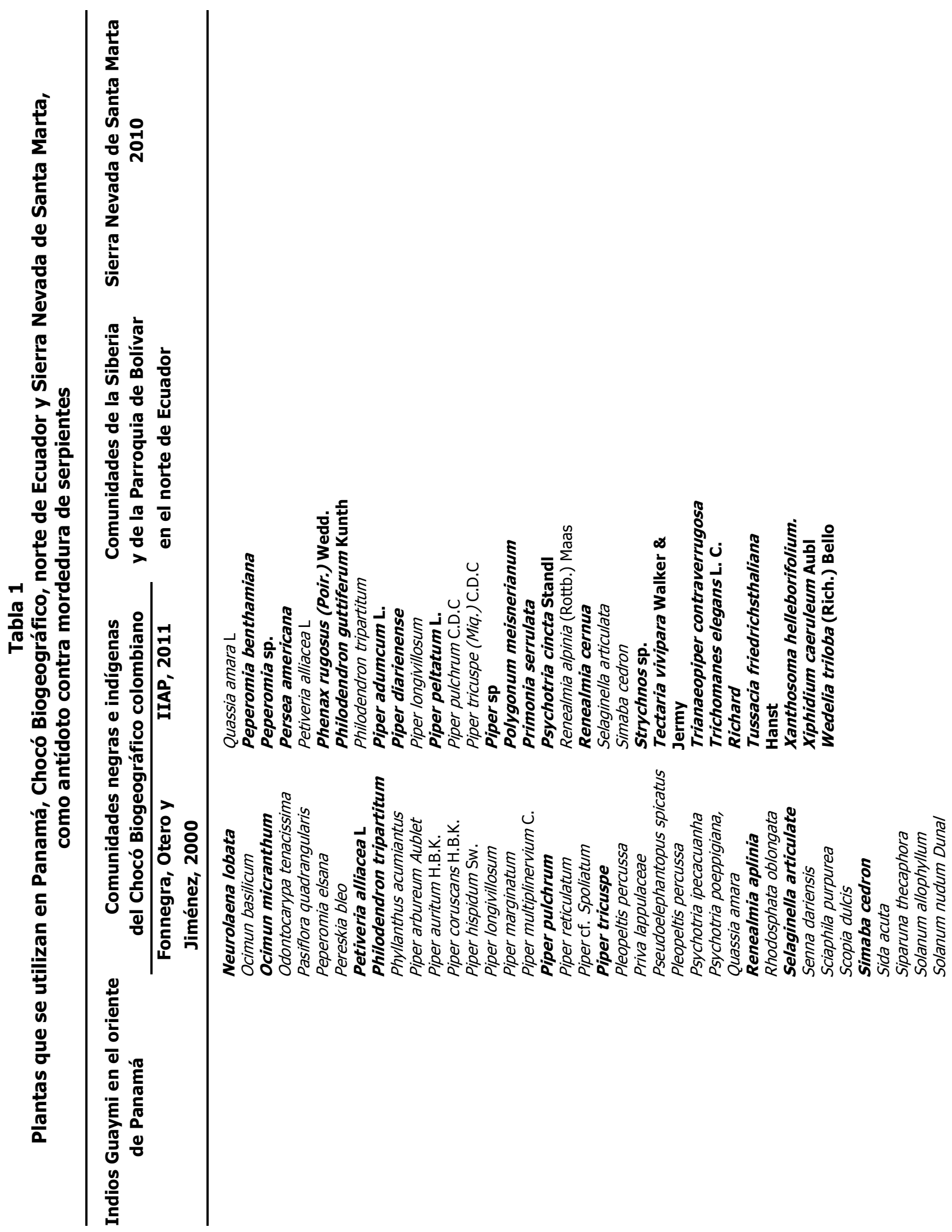




\section{Bioetnia Volumen 9 No 1 (enero-junio), 2012}

[Mikania guaco Humb. y Bunpl; Neurolaena lobata; Wedelia triloba (Rich.) Bello; Adenostemma platyphyllum Cass; Adenostemma lavenia; Clibadium silveltre (Aublet) Baill.; Erechtites valerianaefolia (Wolf.) DC.; Pseudoelephantopus spicatus (Aubl.) Gleas)] y a nivel mundial se agrupan en 34 géneros y 57 especies. Las Euphorbiaceas cuentan a nivel mundial con 23 géneros y 42 especies; en esta familia se reporta una sola especie con uso antiofídico en el Chocó Biogeográfico (Phyllantus acuminatus Vahl). La familia Apocynaceae a nivel mundial se agrupa en 15 géneros y 27 especies, con solo dos especie reportada en el Chocó Biogeográfico (Malouetia isthmica y Allamanda cathartica L). Por otro lado se informa el uso antiofídico a nivel mundial de cerca de 20 especies de Verbenaceas y 3 especies reportadas a nivel del Chocó Biogeográfico (Aegiphila panamensis Mold; Priva lappulaceae (L.) Pers.; Stachytarpheta cayennensis (L.C. Rich) Vahl.).

Las Araceas son una familia muy interesante con hasta 26 especies antiofídicas a nivel mundial, de las cuales 14 se encuentran en el Chocó Biogeográfico representadas por los géneros Anthurium ( $A$. subsignatum, A. cf. Tridigitatu, y A. mormotathum), Dracontium ( $D$. grayumianum, D. loretense, $D$. croati Zhu), Philodendron ( $P$. guttiferum y $P$. tripartitum), Xanthosoma (X. helleborifolium), Alocasia (A. cucullata), Dieffenbachia (D. longisphatha) y Rhodosphata (R. oblongata).

Entre los géneros más significativos, el más abundante es Aristolochiae Aristolochiae, del cual hasta 24 especies se usan tradicionalmente es familia o como remedio contra la mordedura de serpiente en casi todo el mundo (Lópezy Pérez, 2009), Centroamérica (A. arborescens, A. grandiflora, A. maxima, A. odoratissima, A. pentandra), Brasil (A. barbata, A. theriaca), Colombia (A. maxima, A. rigen), India (A. bracteata, $A$. indica, A. longa, A. serpentaria) y sudeste asiático (A. cucurbitifolia, A. debilis), oriente de Panamá (A. chapmaniana, A. constricta, A. pilosa, A. sylvicola), Chocó Biogeográfico (A. trilobata L, A. grandiflora, A. pilosa, A. pfeiferi K) y norte de Ecuador (A. pilosa). De estas especies se utiliza la planta entera y sobre todo sus raíces, y en algunos casos las hojas y el látex. En el caso del Chocó Biogeográfico se usan las hojas y tallos en la preparación de botellas balsámicas dirigidas a la cura y/o alivio de mordedura de serpientes.

Un caso especial es el que muestra la familia Piperaceae que a nivel mundial se reportan 9 especies distribuidas en 2 géneros, mientras que en el Chocó Biogeográfico, se reportan 17 especies Piper si es gé agrupadas en 2 géneros: Piper ( $P$. adumcum, $P$. diarienense, $P$. nero debe ir er longivillosum, $P$. peltatum, $P$. pulchrum y $P$. tricuspe, $P$. arboreum, cursiva $P$. auritum, $P$. coruscan, $P$. hispidium, $P$. marginatum, $P$. multiplinervium, $P$. reticulatum; $P$. cf. spoliatum); Trianaeopiper (T. contraverrugosa), mientras que en el norte de Ecuador se utilizan $(P$. eriopodo y $P$. peltata).

La familia Gesneriaceae representada por 5 géneros (Columnea, Episcia, Primonia, Tussacia y Besleria) y siete especies (Columnea picta, Columnea sp, Episcia dianthiflora, Episcia lilacina y Primonia serrulata, Columnea pulcherrima y Besleria sanguinea) a nivel mundial, además de un nuevo reporte de especie (Tussacia friedrichsthaliana) perteneciente al género Tussacia, todos con presencia en el 


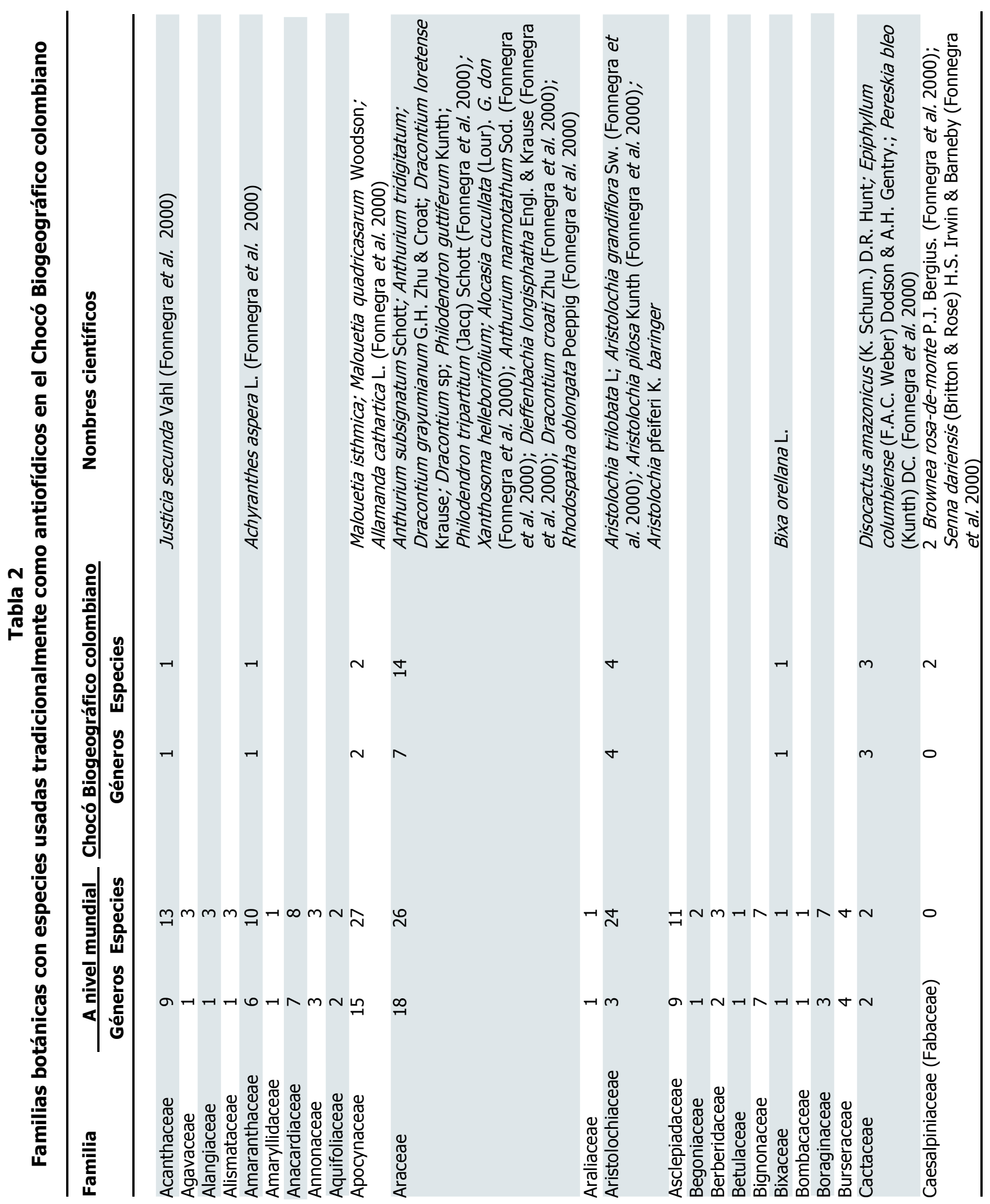


Bioetnia Volumen 9 No 1 (enero-junio), 2012

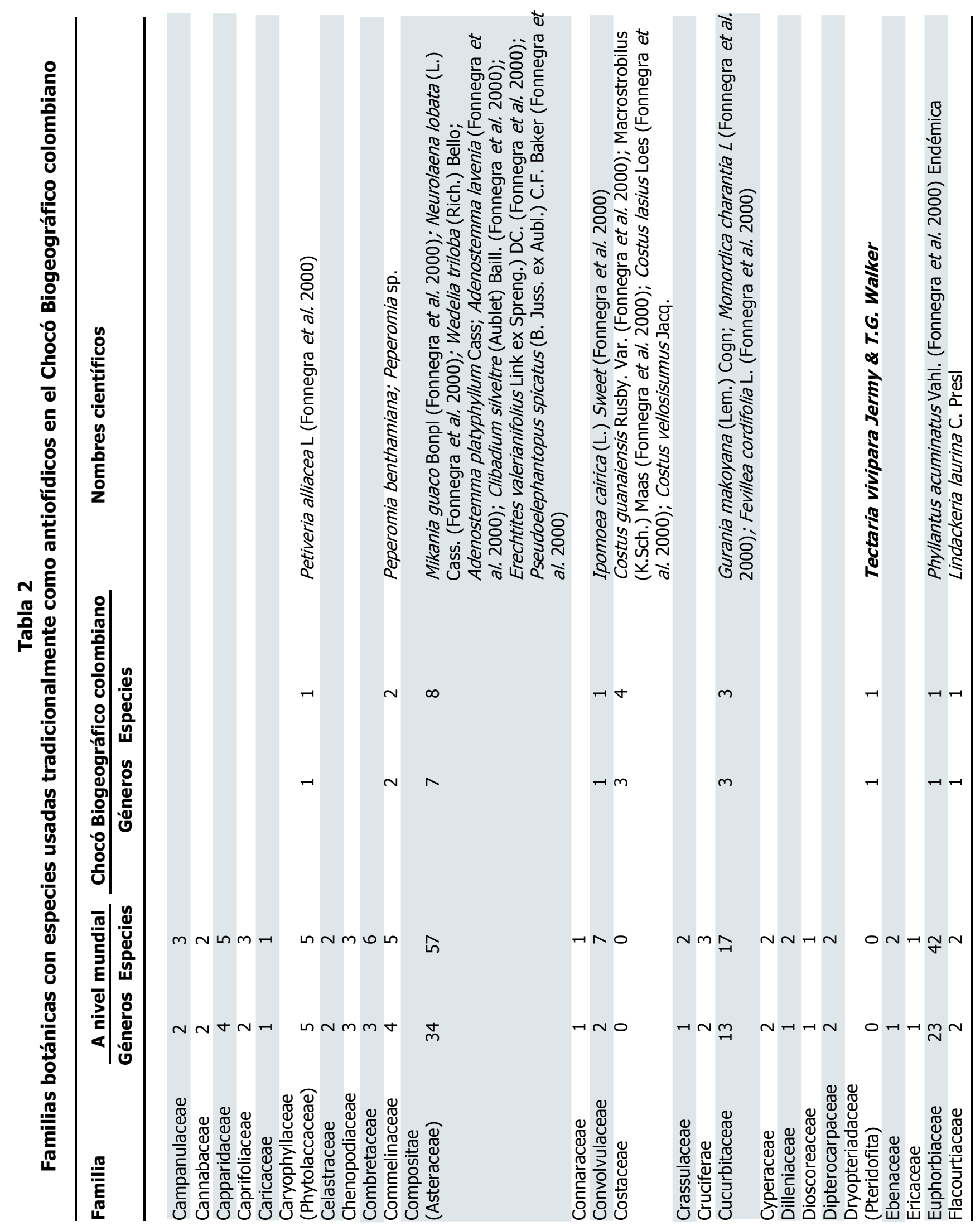




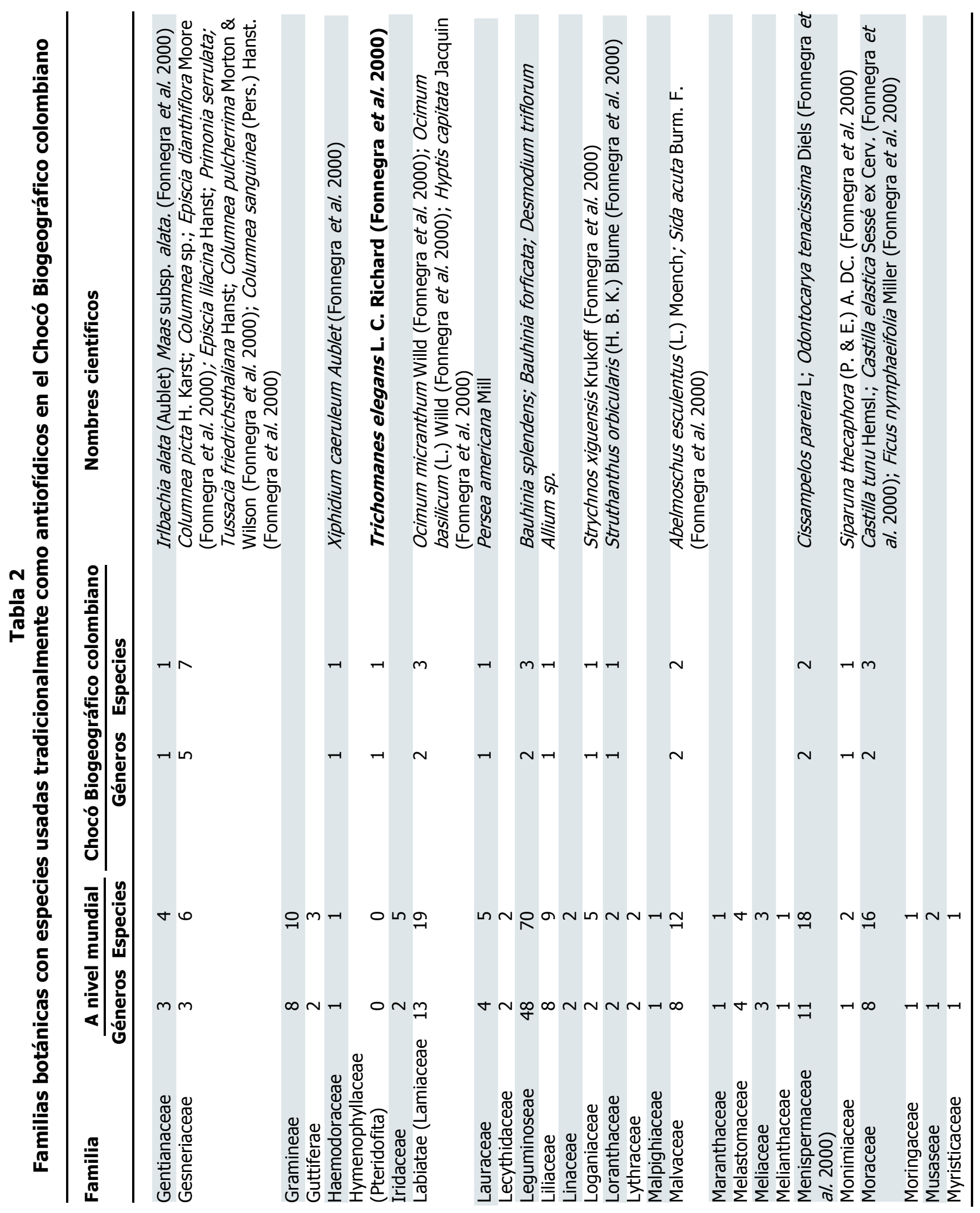


Bioetnia Volumen 9 № 1 (enero-junio), 2012

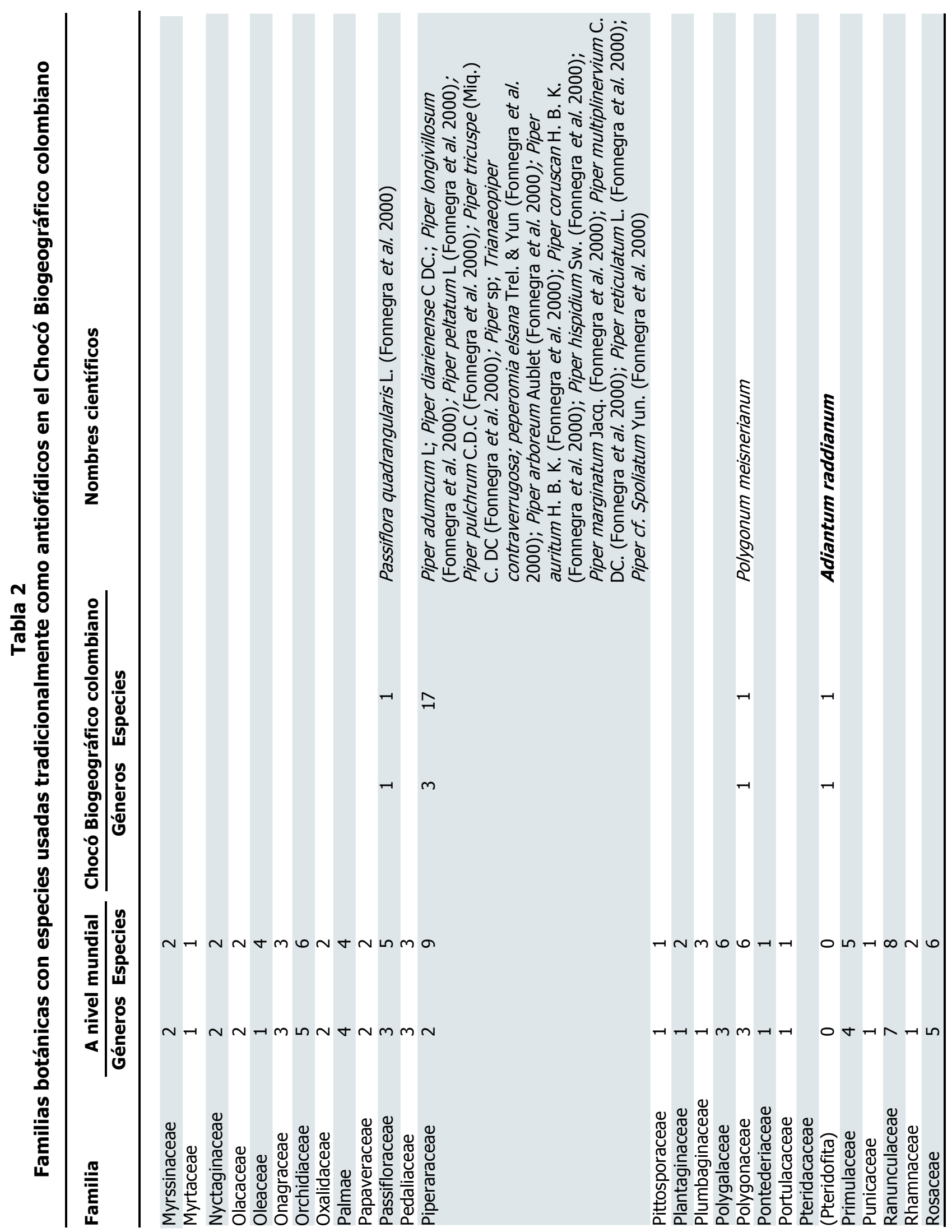




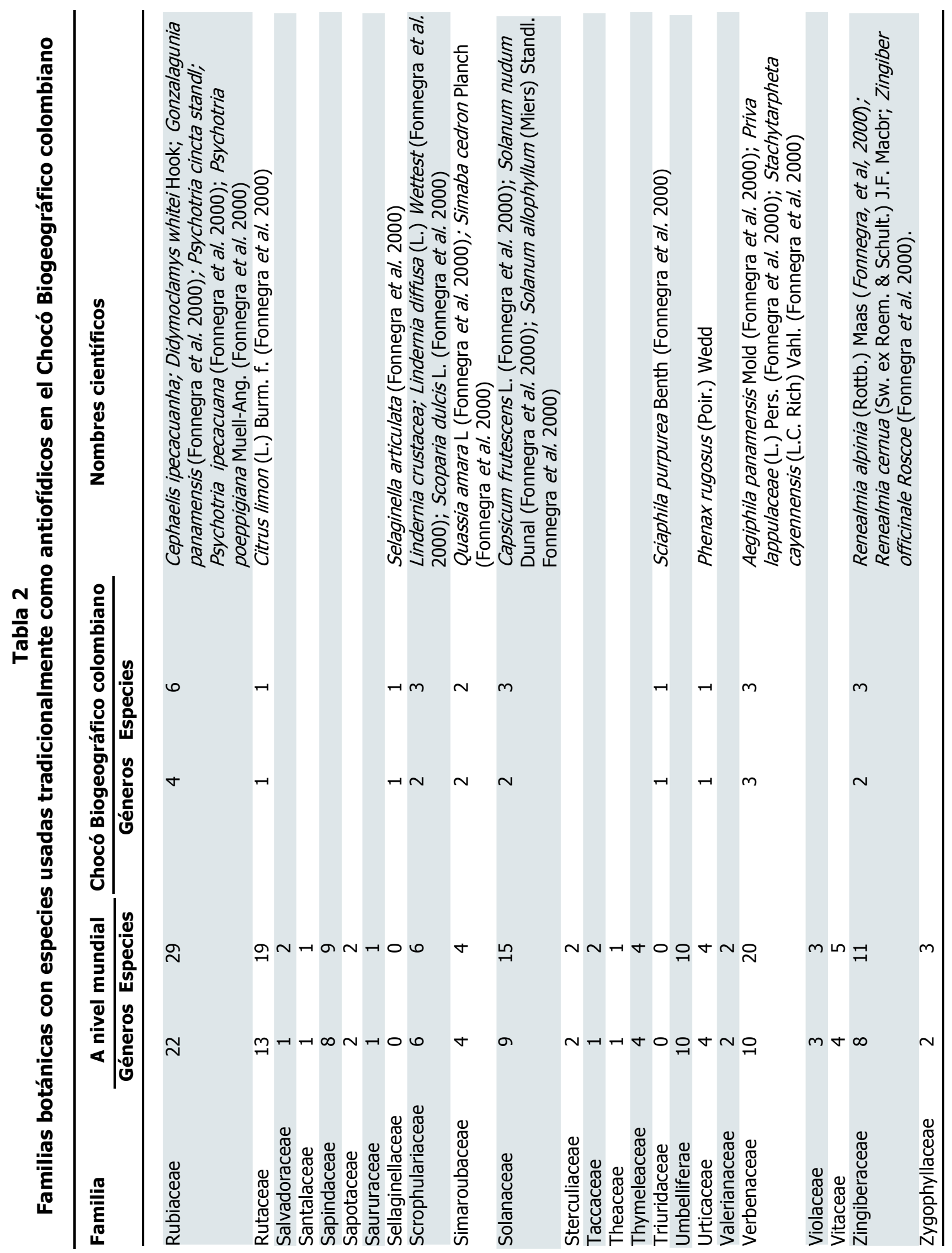




\section{Bioetnia Volumen 9 No 1 (enero-junio), 2012}

Chocó Biogeográfico.

López y Pérez (2009), informan muchas plantas de uso alimenticio, condimentario, colorante u ornamental que se utilizan para combatir los efectos causados por la mordedura de serpientes como mango (Mangifera indica), litchi (Litchi chinensis), azafrán (Crocus sativus), papaya (Carica papaya), garbanzo (Cicer arietinum), nuez moscada (Myristica fragans), pimiento (Capsicum annuum), longán (Euphoria longan), ricino (Ricinus communis), ajo (Allium cepa), cebolla (Allium sativum), batata (Ipomoea batatas), kaki (Diospyros kaki), hojas de alcachofa (Cynara scolymun) en Brasil, adelfa (Nerium oleander) en Oriente Medio, las hojas de la flor de sangre (Asclepias curassavica) en Centroámerica o las de achiote (Bixa Orellana) en la India y Filipinas, las raíces de la mandioca (Manihot esculenta), las semillas del cacao (Theobroma cacao) en Suramérica, coco (Cocos nucifera), y finalmente muchas especies en la India como girasol (Helianthus annus), granado (Punica granatum), vid (Vitis vinifera) y cidro (Citrus medica). Algunos cactus como Opontia dillenii o el alucinógeno peyote (Lophophora williamsii), son antiofídicos reputados en la India y América Central respectivamente como las hojas del cáñamo (Cannabis sativa) y del tabaco (Nicotiana tabacum), o la planta de la adormidera (Papaver somniferum).

Llama la atención que sólo tres de estas plantas de uso alimenticio, colorante u ornamental reportadas por López y Pérez (2009) sean utilizadas para tratar la mordedura de serpientes en el Chocó Biogeográfico: achiote (Bixa orellana) del cual se utiliza la semilla a diferencia del uso de las hojas que se da en la India y Filipinas, cebolla (Allium sativum cepa L.), aunque se reporta el uso de otras especies agrupadas a esta categoría que ampliarían este listado a cuatro especies más: aguacate (Persea americana), albahaca morada (Ocimun micranthum), ají (Capspicum frutescens L.), limón (Citrus limon), granadilla (Pasiflora quadrangularis) (Tabla 2).

Estos resultados comprueban el vasto acervo de conocimientos que poseen los grupos étnicos asentados en el Chocó Biogeográfico colombiano, sobre todo relacionados con plantas medicinales, los cuales se encuentran representado en el uso que sabedores tradicionales, le dan a diferentes plantas medicinales para la cura $\mathrm{y} / \mathrm{o}$ alivio de las afecciones que comúnmente se asociadas con algún tipo de envenenamiento ocasionado por mordedura de serpiente, lo cual es indicativo de que este conocimiento es muy superior al encontrado por diversos estudios de este tipo y realizados con otros grupos étnicos. Por ejemplo, los Colorado, Cayapa y Coaquier (norte de Ecuador) utilizan un conjunto de 40 especies de Gesneriaceas (Kvris, 1986), 11 y 17 especies de Polypodiales y Piperaceas respectivamente (Holm-Nielsen et al., 1983). Los habitantes del sitio La Siberia y de la Parroquia Bolívar en el Ecuador, utilizan 26 especies para tratar la mordedura de serpientes, entre las que se destacan seis Gesneriaceas y tres Piperaceas (Lajones, 2006) (Tabla 1). En las Guayanas se utilizan 42 especies de plantas para tratar la mordedura de serpientes y los indios Guaymi del oeste de Panamá emplean cerca de 11 plantas para este fin (Tabla 1).

En el Pacífico colombiano, se reportan cerca de 105 plantas con algún tipo de uso para tratar la mordedura de serpientes (Fonnegra, Otero y Jiménez, 2000) (Tabla 1); sin embargo, en este estudio se informan 70 etno-especies de plantas medicinales utilizadas por médicos tradicionales y jaibanás sobre todo de la etnia cuna para el tratamiento de la mordedura de serpientes (nombres en negritas, Tabla 1), las cuales 20 etnoespecies hasta el momento no habían sido reportadas para tal fin, lo que amplía a 125 el número de especies de plantas utilizadas por comunidades negras e indígenas para el tratamiento de la mordedura de serpientes en el Chocó Biogeográfico colombiano.

Si se observa la Tabla 1, vemos que en los bosques tropicales que hacen parte de las cuatro zonas descritas en la misma tabla, se presentan algunas coincidencias: A nivel de familias botánicas, las familias Aristolochias, Asteraceas, Simarubaceas, Pasifloraceas, Solanaceas, Commelinaceas, Rubiaceas y Scrophulariaceas son utilizadas en estas cuatro zonas -así como en muchas partes del planeta-, igualmente ocurre con las géneros Aristolochia, Pasiflora y Simaba, los cuales también se usan en estas cuatro zonas. Sin embargo, la única coincidencia es la especie Simaba cedron que se emplea en las cuatro comunidades estudiadas para tratar la mordedura de serpientes.

Una vez revisada la literatura disponible sobre el tema, estamos agregando a la lista 24 especies adicionales, así: Trichomanes elegans, Selaginella articulata, Sciaphila purpurea, Costus guanaiensis; C. lasius; Brownea rasedemonte, Senna dariensis, Piper adumcum, $P$. diarienense; $P$. longivillosum; $P$. peltatum; $P$. pulchrum; $P$. sp; Trianaeopiper contraverrugosa; Peperomia elsana; Piper arboreum; $P$. auritum, $P$. coruscan; $P$. hispidium; $P$. marginatum; $P$. multiplinervium; $P$. reticulatum; $P$. cf. spoliatum.

Además, se informa en esta publicación 3 nuevas plantas que se utilizan exclusivamente por comunidades negras e indígenas del Chocó Biogeográfico colombiano: Tectaria vivipara, Costus vellosisumus, Adiantum raddianum.

Nombre científico: Tectaria vivipara Walker y Jermy

Nombre vulgar: Nido de culebra

Familia: Dryopteridaceae/Pteridofitas

Descripción: Helecho terrestre sin tallo visible. Su fronda consta de foliolos simples y enteros; su folíolo basal tiene un lóbulo alargado. Se encuentra ocasionalmente en el interior del bosque; crece en bancos de arena de quebradas de sitios rocosos.

Parte usada: Hojas. 


\section{Comparación del uso de plantas medicinales para la cura ylo alivio por mordeduras de serpientes. CA. Rentería}

Usos: Se emplea en la medicina tradicional para que las personas no piensen en culebras. Las hojas frescas se utilizan en baños medicinales o se dejan secar a temperatura ambiente para emplearlas en baños de vapor (sahumerios).

Nombre científico: Costus villosissimus

Nombre vulgar: Cañagria

Familia: Costaceae

Descripción: Hierba de 1-2 metros de altura. De tallo erecto de color verde jugoso; hojas alternas longilíneas envainadas al tallo, flores de color amarillo, acompañadas de brácteas verdes triangulares y dispuestas en inflorescencias racimosas termales, usualmente localizadas en zonas húmedas.

Parte usada: Tallos.

Usos: Se utiliza el zumo que se extrae del tallo, el cual se toma para neutralizar el veneno de culebra.

Nombre científico: Adiantum raddiantum

Nombre vulgar: Culantrillo

Familia: Pteridaceae/Pteridofitas

Descripción: Rizoma brevemente reptante, con escamas castañas, enteras a denticuladas, tallos de color púrpura a negros. Hojas de 22 a $45 \mathrm{~cm}$ de largo, arqueadas, distantes, una vez divididas (pinnadas) o dos veces (bipinnadas), cada uno de los folíolos ovado lanceolados, lampiños en ambas superficies, venas libres surcadas. Soros marginales numerosos, oblongos, cubiertos por el envés de la lámina.

Parte usada: Hojas.

Usos: De las hojas, se realiza una cocción con la que se prepara un emplasto, el cual se aplica en compresas en el área afectada por la mordida de la serpiente.

\section{Conclusiones}

A nivel del Chocó Biogeográfico colombiano, a través de diferentes publicaciones se reportan cerca de 105 plantas con algún tipo de uso para tratar la mordedura de serpientes (Otero et al., 2000). Sin embargo, el IIAP a través de diferentes investigaciones y conversaciones con médicos tradicionales en relación con plantas del Chocó Biogeográfico utilizadas para el tratamiento de la mordedura de serpientes tiene información sobre el uso de 70 etnoespecies vegetales usadas para este propósito. Tres de estas etnoespecies de plantas hasta el momento no han sido informadas como agentes antiofídicos, lo que amplía a 125 el número de especies de plantas utilizadas por médicos tradicionales o jaibanás del Chocó Biogeográfico colombiano para el tratamiento de las diferentes afecciones producidas por mordedura de serpientes venenosas.

A nivel del Chocó Biogeográfico colombiano, las especies antiofídicas más representativas corresponde a la familia Piperacea con 17 especies, seguida de la familia de las Araceas con 13 especies. En cuanto a los géneros el más representativo fue el Piper con 17 especies y los géneros Dracontium, Aristolochia y Columea con 4 especies cada uno.

Se reportan tres nuevas etnoespecies de plantas antiofídicas que son utilizadas exclusivamente por comunidades negras e indígenas del Chocó Biogeográfico colombiano: Tectaria vivipara, Costus vellosisumus, Adiantum raddianum.

\section{Recomendaciones}

Existe una fuerte interacción entre los habitantes del Chocó Biogeográfico colombiano y el componente tradicional de nuestros pueblos negros e indígenas, que prefieren utilizar el servicio de jaibanás en el caso de los indígenas, y curanderos o médicos tradicionales en el caso de las comunidades negras, antes de buscar ayuda en hospitales y centros de salud. Por ello es de gran importancia iniciar el estudio de las plantas utilizadas como antídotos contra la mordedura de serpientes en nuestra medicina tradicional. Se debe determinar si dichas plantas o sus extractos son realmente activas en la neutralización de los principales efectos fisiopatológicos de los venenos, con lo cual podría mejorarse el tratamiento de emergencia del accidente ofídico en el país. En caso contrario, es importante también mostrar las limitaciones de estas sustancias para prevenir que el paciente reciba un tratamiento inadecuado con hierbas sin valor curativo.

\section{Literatura citada}

Barranco-Pérez, W., M. Sánchez-Sáenz. 2010. Especies vegetales de uso antiofidico en las estribaciones de la Sierra Nevada de Santa Marta: inventario etnobotánica y evaluación biológica. Tesis de Maestría. Medellín: Universidad Nacional de Colombia.

Campbell, J.A. W.W. Lamar. 1989. The venomous reptiles of Latin America. Ithaca: Cornell University Press. $452 \mathrm{pp}$.

Duke, J. 1998. Phytochemical and ethnobotanical database. URL disponible en: http://www.ars.grin.gov/cgi-bin/duke/ethnobotuse.pl

Firenzouli, F., I. Gori, D, Neri. 2005. Clinical phytotherapy: opportunities and problematic. Ann Ist Supert Sanita. 41 (1): 27-33.

García, E. 1896. Los ofidios venenosos del Cauca. Métodos empíricos y racionales empleados contra los accidentes producidos por la mordedura de ofidios venenosos. París: Editorial de Bouret. p. 58-102.

Holm-Nielsen L., L.P. Kvist, M. Aguavil 1983. Las investigaciones etnobotánicas entre los Colorados y los Cayapas. Informe preliminar. MAE. 3: 89-116.

Houghton P. J, I.M. Osibogun. 1993. Flowering plants used against snakebite. J Etnopharmacol. 39: 1-29.

Houghton P.J., I.M. Osibogun. 1993. Flowering plants used against snakebite. J Ethnopharmacol. 39: 1-29.

Joly, L. G., S. Guerra, R. Séptimo, P. N. Solís, M. Correa, M. Gupta, et al. 1987. Ethnobotanical inventory of medicinal plants used by the Guaymi Indiams in Western Panamá. Part I. J Etnhopharmacol. 20: 145-71.

Kvist, L.P. 1986. Gesneriads y snake bite. Gloxinian. 36 (1): 8-13.

Lajones, A. 2006. Plantas medicinales utilizadas por los habitantes del sitio La Siberia y de la Parroquia Bolivar. Guayaquil: EcoCostas.

Li, Y., Y. Ohizumi. 2004. Search for constituents with neurotrophic factor potentiating activity from the medicinal plants of Paraguay and Thailand. Yakugaku Zasshi. 124 (7): 417-24. 


\section{Bioetnia Volumen 9 No 1 (enero-junio), 2012}

López, J.A., J. Pérez. 2009. Plantas alexitéricas: antídotos vegetales contra las picaduras de serpientes venenosas. Med Nat. 3 ( 1): 17-24.

Martin, G.J. 2000. Etnobotánica. Manual de métodos: Manuales de conservación. Serie Pueblos y Plantas 1. Montevideo: World Wildlife Fund, Organización de las Naciones Unidas para la Educación, la Ciencia y la Cultura y Royal Botanic Gardens.

Martz, W. 1992. Plants with a reputation against snakebite. Toxicon. 30: 113142.

Mashkar. K.S., J.F. Caius. 1931. Indian plant remedies used in snakebite. Indian J Med Res. (Suppl.) 19: 96.

Mors, W.B. 1991. Plants active against snake bite. En: Wagner H., N. R. Farnsworth (eds.). Economic and medicinal plants research. Vol, 5, Plants and traditional medicine. New York: Academic Press. p. 353-73.

Noriega-Trejo R. 1993. Mordeduras de serpientes venenosas y plantas medicinales: Una visión general. Simposio sobre Herbolaria Aplicada en
Veterinaria. Noviembre 10-12. Xochimilco: Universidad Autónoma Metropolitana.

Otero, P. R., R. Fonnegra, S. L. Jiménez (2000). Plantas utilizadas contra mordeduras de serpientes en Antioquia y Chocó, Colombia. Medellín: Ediciones Grandacolor.

Reyes R., M. Jiménez. (1995). Química de las plantas alexíteras. Interciencia. 20: $257-64$

Reyes-Chilpa, R., F. Gómez-Garibay, L. Quijano, G. A. Magos-Guerrero, T. Ríos-Castillo 1994. Preliminary results on the protective effect of (-)edunol a pterocarpan from Brongniartia podalyrioides (Leguminosae) against Bothrops atrox venom in mice. J Ethnopharmacol. 42:199-203.

Rizzini, C.T., W.B. Mors, N. Alvares-Pereira. 1988. Plantas brasileiras tidas como ativas contra peçonhas animais, especialmente venenos de cobras. Rev Bras Farm. 69 (4): 82-6.

Tuxill J., G.P. Nabhan 1998. Plants and protected areas. A guide to in situ management. Cheltenham: Stanley Thornes. 